\title{
Tissue-Adaptive Materials with Independently Regulated Modulus and Transition Temperature
}

\author{
Daixuan Zhang, Erfan Dashtimoghadam, Farahnaz Fahimipour, Xiaobo Hu, \\ Qiaoxi Li, Egor A. Bersenev, Dimitri A. Ivanov, Mohammad Vatankhah- \\ Varnoosfaderani, and Sergei S. Sheiko*
}

\author{
D. Zhang, Dr. Q. Li \\ Department of Applied Physical Sciences \\ University of North Carolina at Chapel Hill \\ Chapel Hill, NC 27599-3050, USA \\ Dr. E. Dashtimoghadam, Dr. F. Fahimipour, Dr. X. Hu \\ Dr. M. Vatankhah-Varnoosfaderani, Prof. S. S. Sheiko \\ Department of Chemistry \\ University of North Carolina at Chapel Hill \\ Chapel Hill, NC 27599-3290, USA \\ E-mail: sergei@email.unc.edu \\ E. A. Bersenev \\ Moscow Institute of Physics and Technology \\ Institutsky Lane 9, Dolgoprudny, Moscow region 141700, Russian Federation \\ E. A. Bersenev, Prof. D. A. Ivanov \\ Institute of Problems of Chemical Physics \\ Russian Academy of Sciences \\ Chernogolovka 142432, Russia \\ Prof. D. A. Ivanov \\ Institut de Sciences des Matériaux de Mulhouse-IS2M \\ CNRS UMR 7361, 15, rue Jean Starcky, Mulhouse F-68057, France \\ Prof. D. A. Ivanov \\ Lomonosov Moscow State University \\ Moscow 119991, Russian Federation \\ The ORCID identification number(s) for the author(s) of this article \\ can be found under https://doi.org/10.1002/adma.202005314 \\ DOI: 10.1002/adma.202005314
}

The ability of living species to transition between rigid and flexible shapes represents one of their survival mechanisms, which has been adopted by various human technologies. Such transition is especially desired in medical devices as rigidity facilitates the implantation process, while flexibility and softness favor biocompatibility with surrounding tissue. Traditional thermoplastics cannot match soft tissue mechanics, while gels leach into the body and alter their properties over time. Here, a single-component system with an unprecedented drop of Young's modulus by up to six orders of magnitude from the GPa to $\mathrm{kPa}$ level at a controlled temperature within $28-43{ }^{\circ} \mathrm{C}$ is demonstrated. This approach is based on brush-like polymer networks with crystallizable side chains, e.g., poly(valerolactone), affording independent control of melting temperature and Young's modulus by concurrently altering side chain length and crosslink density. Softening down to the tissue level at the physiological temperature allows the design of tissueadaptive implants that can be inserted as rigid devices followed by matching the surrounding tissue mechanics at body temperature. This transition also enables thermally triggered release of embedded drugs for anti-inflammatory treatment.

Various types of clinical complications may emerge when a medical device (catheter, microelectrode, or microneedle patch) is deployed to the human body. It has been widely recognized that mechanical mismatch between an implant and a host tissue is one of the leading factors for adverse effects such as irritation and inflammation, which is evident in orthopedic,[1] neural,[2,3] and reconstructive implants.[4] It is generally desired that an implant material precisely matches Young's modulus of the surrounding tissue that may range between $E \approx 102$ $\mathrm{Pa}$ of supersoft brain and adipose tissues to $E \approx 105 \mathrm{~Pa}$ of muscle and skin. However, forceful implantation of biomedical devices such as piercing, insertion or twisting impose significant mechanical stresses on the device.[5-8] Therefore a certain rigidity is desired to prevent device failure, minimize tissue damage, and simplify storage and handling procedures.[9,10] This presents an oxymoronic challenge for an ideal implant: designing an adaptive material that 
readily penetrates tissue, but transitions into a tissue-soft implant upon insertion into the body (Figure 1A). Thermoplastics are one of the most commonly used materials possessing a welldefined transition between hard and soft states with Young's moduli of Ehard $\approx 1 \mathrm{GPa}$ and Esoft $\approx 1 \mathrm{MPa}$, respectively (Figure 1B). However, conventional thermoplastic materials have two problems: i) Esoft $\approx 1 \mathrm{MPa}$ is significantly higher than soft tissue modulus ranging within 102$105 \mathrm{~Pa}$ and ii) softening (melting or glass) transition temperature of high molecular weight polymers cannot be tuned without altering their chemical composition. It is even more challenging to adjust the mechanical and thermal properties independently of each other. The softness issue alone could be resolved by using different types of bioinspired polymeric gels.[14-17] However, not only it is difficult to prepare gels with a sharp GPa-tokPa transition (Figure 1C), their solvent fraction instigates uncontrolled leaching inside the body,[13] which causes various health risks. The second problem can be addressed via copolymerization of monomers with different melting temperatures.[18-20] However, this involves chemical composition and biocompatibility variation and yet, copolymer modulus is limited by chain entanglements to >105 $\mathrm{Pa} .[21,22]$

A

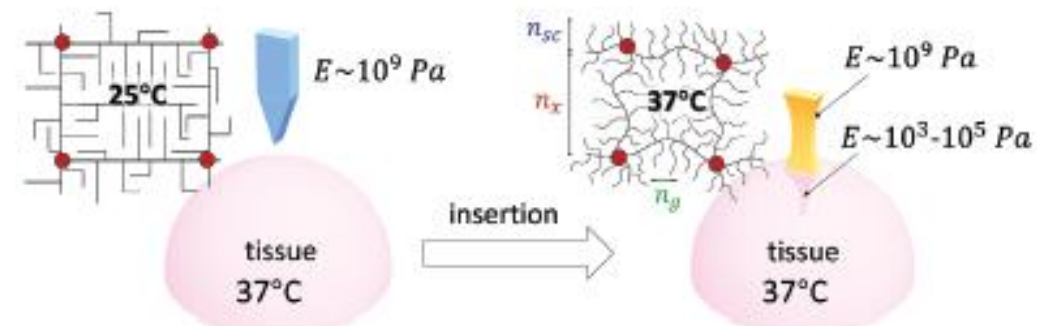

B
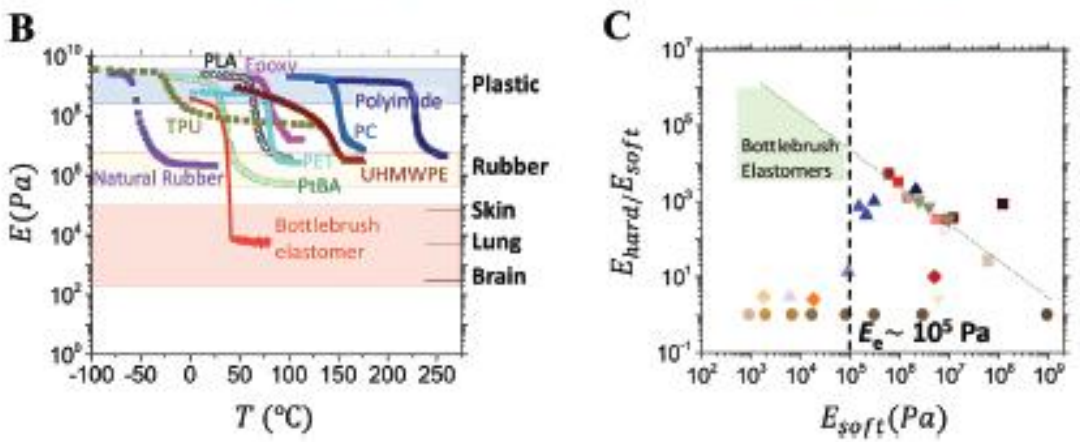

Figure 1. Hard-to-soft transition. A) Schematic representation of a tissue-adaptive implant. At a room temperature of $25^{\circ} \mathrm{C}$, an implant is rigid, easily penetrating tissue followed by softening at a body temperature of $37^{\circ} \mathrm{C}$ to match the mechanics of the surrounding tissue $\left.(\approx 102-105 \mathrm{~Pa}) . \mathrm{B}\right)$ Many polymeric materials undergo a sharp modulus decrease upon melting from a hard-state modulus of Ehard $\approx 1$ Gpa ("Plastic" bar) to a soft-state modulus of Esoft $\approx 1$ Mpa ("Rubber" bar). Their Esoft cannot go below $\approx 105-106 \mathrm{~Pa}$ due to chain entanglements. In contrast, architecturally disentangled bottlebrush elastomers with crystallizable side chains allow a much greater modulus drop from the Gpa to kPa level ("Tissue" bar). Furthermore, the brush-like architecture allows mimicking the mechanics of specific tissues such as skin, lung, and brain[11] by varying the architectural triplet $[n \mathrm{sc}, n \mathrm{~g}, n x]$ : degrees of polymerization (DP) of the side chains ( $n \mathrm{sc})$, of the spacer between neighboring side chains ( $n \mathrm{~g})$, and of the strand backbone $(n x) .[12,13]$ C) Mapping conventional materials (including thermoplastics, gels, and tissues) by $\log -\log$ plotting their modulus drop (Ehard/Esoft) against their Esoft. Herein, Ehard and Esoft correspond to Young's moduli measured before and after a softening transition under condition specified. Most thermoplastics (squares) and thermosets (downward triangles) have similar Ehard $\approx 1 \mathrm{Gpa}$ and, therefore, fall on the same line with -1 slope. Specifically, Esoft values of thermoplastics correspond to a rubbery plateau modulus due to chain entanglements. For thermosets, Esoft corresponds to the Young's modulus of a polymer network controlled by 
crosslink density. In both systems, Esoft is measure above glass and melting transitions at specific temperatures indicated.

As shown, both systems demonstrate a lower limit of $\approx 105-106 \mathrm{~Pa}$ which falls short from reaching tissue-like softness (103-105 Pa). Synthetic gels (upright triangles) and biological materials (circles and diamonds) can reach below this limit, but their softening transition is either marginal or nonexistent. With bottlebrush polymers (green polygon), the polymer trend can be extended below the 105 limit, yielding record high modulus drop (104-106 times) and tissue mimicking softness $(103-105 \mathrm{~Pa})$. Note that this plot is not bound to a particular type of transition, rather offers comparison between bottlebrush elastomers with a wide variety of softening systems.

Both problems can be alleviated by employing bottlebrush polymer architecture (Figure 1A), which promotes disentanglement of network strand and thus allows modulus reduction down to $102 \mathrm{~Pa}$ without adding solvent.[12,13,21] To create a significant hard-to-soft transition within a singlecomponent system, we introduce bottlebrush elastomers with crystallizable sidechains[23-25] that enable architectural control over both the Young's modulus $(E)$ and melting temperature $(T \mathrm{~m})$. In the crystalline state $(T<T \mathrm{~m})$, these materials are hard with Ehard similar to that of other crystalline polymers (108 to $109 \mathrm{~Pa})$. Upon melting $(T>T \mathrm{~m})$, they convert to brushlike elastomers with the modulus dropping by four to six orders of magnitude, which significantly exceeds the conventional $10 \sim 103 \times$ modulus drop demonstrated by commodity plastics and biological materials (Figure 1B,C). Their Tm and Esoft can be respectively tuned within $28-43{ }^{\circ} \mathrm{C}$ and $103-105 \mathrm{~Pa}$ by controlling brush-like network architecture within a singlecomponent non-leaching system, which makes them suitable for biomedical applications.

Furthermore, due to the significant difference in diffusivity between semicrystalline hard and rubbery supersoft states, these materials can be used as thermally triggered antiinflammatory drug delivery systems. We synthesize a series of bottlebrush elastomers with crystallizable poly(valerolactone) (PVL) side chains by graftingthrough copolymerization of PVLmethacrylate (MA) macromonomers with a degree of polymerization (DP) of $n \mathrm{sc}=6-14$ and difunctional PVLdiMA crosslinkers with a DP of 35. The macromonomers (1) and crosslinkers (2) are synthesized by ring-opening polymerization providing accurate control over nsc, which defines the melting temperature (Scheme 1). The macromonomers and crosslinkers are then copolymerized via free-radical polymerization inside a glass mold to prepare covalently crosslinked elastomers (3) as thin films of a well-defined thickness of $\approx 1 \mathrm{~mm}$. By varying the

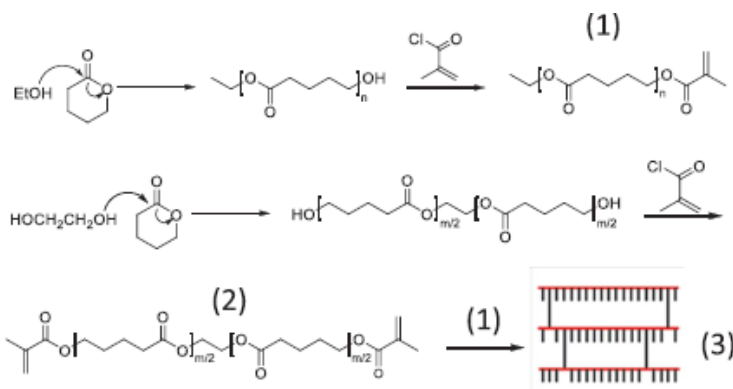

Scheme 1. Synthesis of PVL bottlebrush elastomers.

copolymerization ratio, this synthetic methodology allows accurate control over DP of bottlebrush network strands $(n x)$ and hence crosslink density.[12] The synthetic control over $n$ sc and $n x$ allows for independently tuning thermal and mechanical properties of thermoplastic elastomers. Table 1 shows differential scanning calorimetry (DSC) data of PVL brush elastomers and corresponding macromonomers. Compared to linear macromonomers, bottlebrush networks with the same $n$ sc demonstrate significantly lower degree of crystallinity $(X \mathrm{c})$, lower melting and crystallization temperatures $(T \mathrm{~m}$ and $T \mathrm{c})$, and a wider crystallization 
range $\Delta T=T \mathrm{~m}-T \mathrm{c}$.[26] These effects are ascribed to steric hindrance of densely grafted side chains $[27,28]$ as evidenced by the absence of spherulitic morphology typically

Table 1. Thermal properties of selected PVL bottlebrush elastomers.

\begin{tabular}{|c|c|c|c|c|c|c|c|c|}
\hline \multirow[t]{2}{*}{$m_{2 x^{*}}$} & $n_{y}^{60}$ & $T_{n}{ }^{d}$ & $\Delta H_{m}{ }^{0}$ & $T_{c} e^{d i}$ & $\Delta \mathrm{H}^{\mathrm{\phi}}$ & $x_{2}^{4}$ & $x_{\varepsilon}^{n}$ & $h^{n}$ \\
\hline & & $\mathrm{FC}$ & {$\left[\mathrm{E}^{-1}\right]$} & $\mathrm{FC}$ & {$\left[g^{-1}\right]$} & DSC & XRD & {$[\mathrm{nm}]$} \\
\hline \multicolumn{9}{|c|}{ Macromonomes } \\
\hline 8 & NA & 38.1 & 85.9 & 18.4 & 85.0 & 0.07 & & \\
\hline 10 & NA & 45.1 & 800 & 24.2 & 817 & 0.45 & & \\
\hline 12 & Na & 47 & 85.9 & 26.6 & 84.3 & 0.87 & & \\
\hline 14 & NA & 46.5 & 833 & 26.7 & 807 & 0.46 & & \\
\hline \multicolumn{9}{|c|}{ Bottlebrushes } \\
\hline 6 & 50 & 27.5 & 39.2 & -10.5 & 39.6 & 0.22 & 0.21 & 4.3 \\
\hline 8 & 50 & 33.5 & 45.3 & -9.9 & 45.2 & 0.26 & 0.31 & 4.8 \\
\hline 10 & 50 & 40.0 & 52.1 & 1.9 & 51.6 & 0.29 & 0.35 & 3.5 \\
\hline 6 & 100 & 29.8 & 39.4 & -12.3 & y.8 & 0.22 & NA & $\mathrm{Na}$ \\
\hline 8 & 100 & 324 & 45.9 & -10.9 & 4.1 & 0.26 & NA & Na \\
\hline 10 & 100 & 40.0 & 50.3 & -1.0 & 50.5 & 0.28 & NA & Na \\
\hline 12 & 100 & 427 & 55.3 & 8.0 & 55.3 & 0.31 & NA & Na \\
\hline 14 & 100 & 43.1 & 59.9 & 7.4 & 56.9 & 0.33 & NA & Na \\
\hline 8 & 200 & 31.3 & 46.8 & -10.8 & 43.9 & 0.26 & 0.21 & $\mathrm{Na}$ \\
\hline 10 & 200 & 41.1 & 51.0 & -0.7 & 50.3 & 0.28 & NA & $\mathrm{Na}$ \\
\hline 10 & 400 & 38.0 & 50.9 & -2.34 & 50.0 & 0.28 & NA & $\mathrm{Na}$ \\
\hline
\end{tabular}

Table 2. Mechanical properties of selected PVL bottlebrush elastomers.

\begin{tabular}{|c|c|c|c|c|c|c|c|c|c|}
\hline$\underline{n_{x s}}$ & $n_{3}$ & 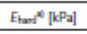 & $E_{z a t} x^{*}[\mathrm{kPa}]$ & $\left.E^{\mathrm{b} \mid \mathrm{kPa}}\right]$ & $E_{0}{ }_{0}^{4}\left[\mathrm{kPa}_{2}\right]$ & $p^{\phi 1}$ & $\lambda_{\operatorname{mas}}$ & $\sigma_{n x^{4}}\left[k_{P} P_{2}\right]$ & $E_{y} c^{4}\left[\mathrm{kP} \mathrm{P}_{2}\right]$ \\
\hline 6 & 50 & $1.52 \times 10^{5}$ & 88.6 & 68.4 & 84.4 & 0.14 & 2.01 & 17 & $\mathrm{Na}^{0}$ \\
\hline 8 & 50 & $2.14 \times 10^{5}$ & 79.5 & 54.1 & 7.3 & 0.18 & 2.25 & 156 & Na \\
\hline 10 & 50 & $3.05 \times 10^{5}$ & 61.9 & 45.5 & 65.0 & 0.22 & 2.18 & 133 & Na \\
\hline 6 & 100 & $1.45 \times 10^{5}$ & 45.1 & 41.6 & 55.2 & 0.19 & 2.1 & $98 \mathrm{~J}$ & 520 \\
\hline 8 & 100 & $260 \times 10^{5}$ & 997 & 32.8 & 4.8 & 0.23 & 3.37 & 576 & 76.9 \\
\hline 10 & 100 & $2.87 \times 10^{5}$ & 33.5 & 21.6 & 32.6 & 0.25 & 278 & 192 & 289 \\
\hline 12 & 100 & $4.08 \times 10^{5}$ & 31.0 & $\nabla .4$ & $2 . A$ & 0.7 & 2 & 40.4 & $9.13 \times 10^{4}$ \\
\hline 14 & 100 & $4.20 \times 10^{5}$ & 18.4 & 10.5 & עJ & 0.30 & 2.13 & 3.3 & $237 \times 10^{5}$ \\
\hline 8 & 200 & $2.14 \times 10^{5}$ & 24.6 & 22.2 & 35.7 & 0.28 & 3.31 & 556 & $\mathrm{Na}$ \\
\hline 10 & 200 & $282 \times 10^{5}$ & 11.3 & 4.2 & 6.8 & 0.28 & 2.95 & 59.3 & $\mathrm{NA}$ \\
\hline 10 & 400 & $230 \times 10^{5}$ & 6.0 & 1.6 & 27 & 0.30 & 3.74 & 90.9 & Na \\
\hline
\end{tabular}

a)Ehard and Esoft obtained as storage modulus at hard state ( $T \square \square 20 \square \mathrm{C}$ ) and soft state ( $T$ $\square \square 60 \square \mathrm{C}$ ) measured by dynamic mechanical analysis (DMA) with heating rate of $1 \square \mathrm{C}$ min-1 and $0.05 \% / 5 \%$ oscillation strain rate, respectively. Additional tests validate the consistency of modulus measured by oscillatory and tensile tests; b)Structural modulus $(E)$ and firmness parameter $(\beta)$ obtained by fitting tensile true stress-elongation curves measured at soft state ( $T$ $\square \square 60 \square \mathrm{C}$ ) (Figure 2E).
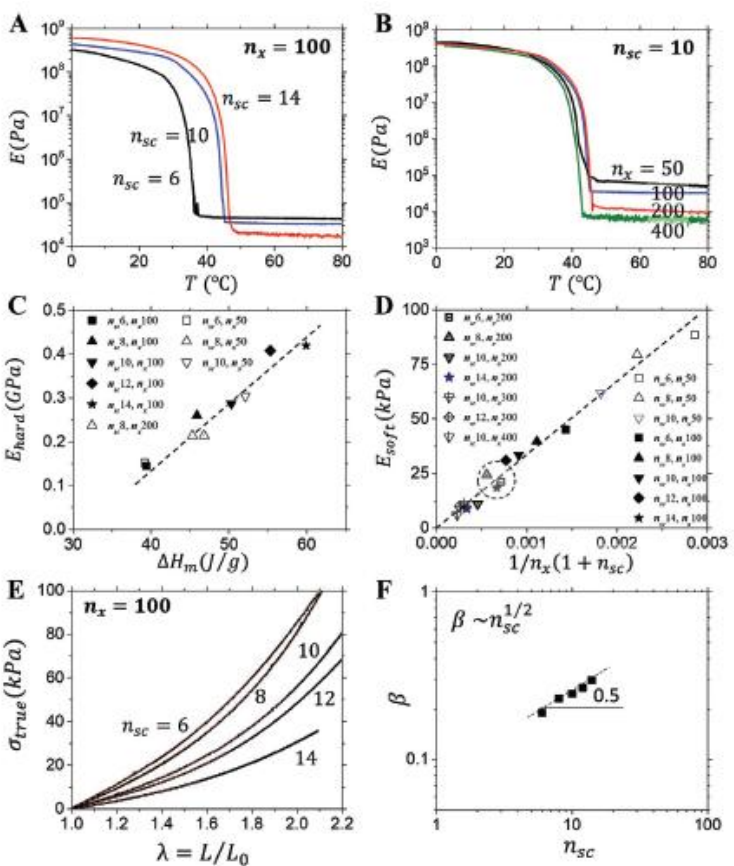

Figure 2. Dual property control. A,B) Melting of brush-like elastomers with crystallizable PVL side chains demonstrates approximately four orders modulus drop in the physiological 
temperature range as measured by DMA (1 $\square \mathrm{C} \mathrm{min}-1)$. A) An increase of side chain length $(\approx n \mathrm{sc})$ results in multiple effects, including increasing Ehard, decreasing Esoft, and increasing $T \mathrm{~m}$. B) Degree of polymerization of network strands $(n x)$ largely affects network softness at $T$ $\square \square T \mathrm{~m}$. Insignificant variation of $T \mathrm{~m}$ is due to thermal history of sample crystallization. C) In the hard state ( $T \square \square 20 \square \mathrm{C}$ ), Ehard increases with the degree of crystallinity (Table 1). D) In the soft state ( $T \square \square 60 \square \mathrm{C}$ ), the Young's modulus (Esoft) increases linearly with crosslink density $\approx \square 1 / n x(1 \square \square n \mathrm{sc})$. The dashed circle designates architectures with a similar crosslink density. E) True stress-elongation curves measured at $60 \square \mathrm{C}$ upon uniaxial extension of PVL bottlebrush elastomers with different $n$ sc at a given $n x \square \square 100$. F) Firmness parameter $\beta$ of the samples from E increases with side chain length in agreement with the theoretically predicted $\square \square \square \square n \mathrm{sc} .[31]$

frequency sweep with a rotational rheometer. Similar to modulus (Figure 2D), nsc affects elastomer firmness in agreement with the theoretically predicted $\square \square \square \square n s c$ (Figure 2F).[30] Collectively, the Figure 2 data demonstrate the ability of PVL bottlebrush elastomers to independently control their softening temperature $(T \mathrm{~m})$, softness (Esoft), and firmness $(\beta)$ by concomitantly varying $n$ sc and $n x$. For example, two different elastomers such as $n$ sc $\square \square 8, n x$

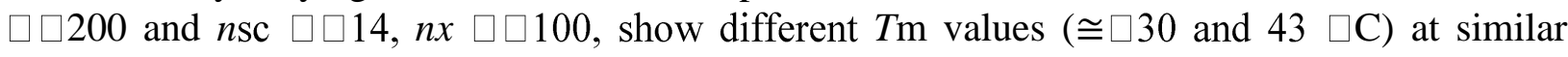
Esoft $\cong \square 20 \mathrm{kPa}$ as their crosslink densities $\approx \square 1 / n x(n \mathrm{sc} \square \square 1)$ are nearly the same (dashed circle in Figure 2D).

Inflammation is one of the notorious complications associated with biomedical devices,[33] including dental,[34] and reconstructive implants.[35] Concurrently with transitioning to tissuemimetic softness, melting of semicrystalline bottlebrush elastomers permits release of imbedded small molecules, e.g., drugs. To demonstrate this capability, a strip of PVL bottlebrush elastomer loaded with acetaminophen, one of the common anti-inflammation drugs, was inserted into a tissue-imitating polyacrylamide hydrogel chromatically responsive to this particular drug (Figure 3). At room temperature, the hard "implant" shows no visible

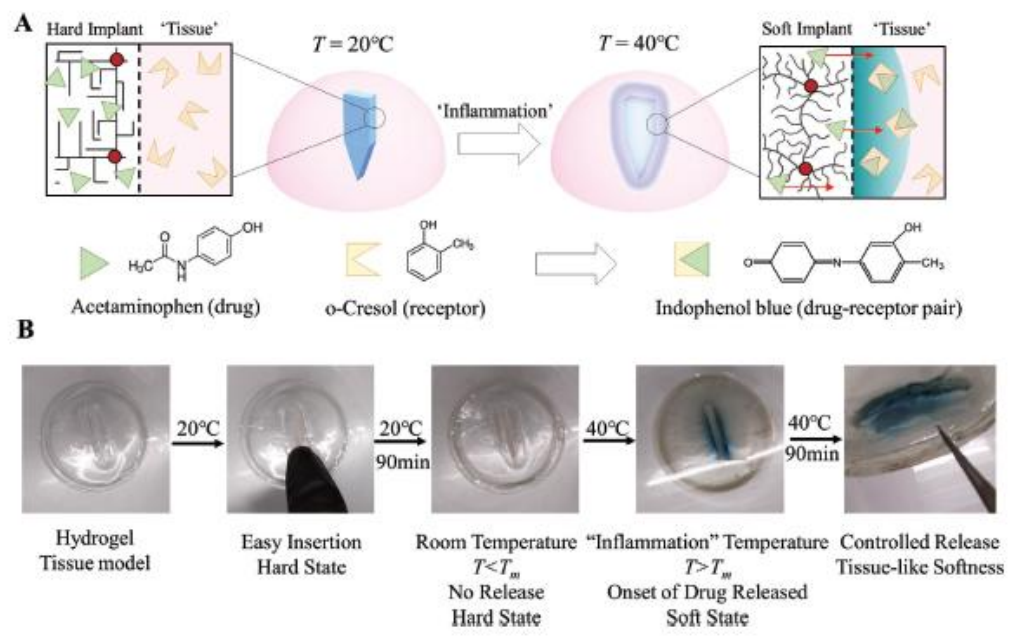

Figure 3. Imitated implant insertion and in situ drug release. A) Schematics of an imitated drug release scenario, where increased temperature triggers the release of drug molecules followed by reaction with receptor molecules resulting in a visual indication. B) Polyacrylamide hydrogel (82 wt $\%$ water) was used as a tissue model loaded with $5 \mathrm{wt} \%$ water solution of o-Cresol molecules (acetaminophen indicators). An "implant" was made out of a PVL bottlebrush elastomer ( $n$ sc $\square \square 10, n x \square \square 50$ ) and loaded with acetaminophen (in $0.1 \mathrm{wt} \%$ water solution). At room temperature (RT), a hard "implant" is inserted into a hydrogel cavity followed by 90 min incubation at RT with no visual indications. Upon increasing temperature to $40 \square \mathrm{C}$, an onset of 
blue coloration is observed around the "implant." During $90 \mathrm{~min}$ at $40 \square \mathrm{C}$, the coloration progressively enhances while the PVL implant remains clear and supersoft. As temperature starts rising and reaches $40 \square \mathrm{C}$, the transition from hard to supersoft state triggers drug release into the surrounding gel resulting in distinct color variation. Therefore, this device enables reactively decreasing inflammation by releasing alleviating drugs under elevated physiological temperature.

In parallel, we conducted a 3 month hydrolytic degradation test of PVL bottlebrush elastomers in a physiological solution under $37 \square \mathrm{C}$ showing no measurable swelling or degradation, which was consistent with long-term stability studies of linear PVL/PCL[36] Ruling out surface erosion, the release of embedded drug happens exclusively through the amorphous phase with its rate dependent on crystallinity, which is controlled by $n$ sc.[37-39] To quantify the $n$ sc dependence of the release rate, a series of PVL bottlebrush elastomers with different $n \mathrm{sc}$ and same $n x \square \square 50$ were loaded with $0.1 \mathrm{wt} \%$ of rhodamine-B which has similar solubility to many small molecule drugs and a distinct absorption peak at $550 \mathrm{~nm}$. At low temperature $(T \square \square 15$ $\square$ C), all three samples demonstrate slow release with a higher release rate for the $n$ sc $\square \square 6$ and 8 samples that possess lower crystallinity

(Figure 4A). At partial melting ( $T \square \square 25 \square \mathrm{C}$ ), an increase in release rate is observed, with greater significance for $n \mathrm{sc} \square \square 6$ and 8 samples due to their lower melting points (Figure 4B). When completely molten ( $T \square \square 37 \square \mathrm{C}$ ), all three samples show initially very similar release profiles followed by deviation at longer times (Figure 4C). Bottlebrush elastomers with longer side chains release overall smaller fraction of rhodamine-B suggesting possible entrapment of small molecules. For thin films (thickness $h \ll$ lateral size), diffusion coefficient $D$ can be deduced by fitting the release curves with the following equation 4 where $M t$ and $M \infty \square$ are amount of drug released at time $t$ and $t$ [40] Plotting $D$ as a function of incubation temperature shows a characteristic S-shaped increase of the release rate with a stronger contrast between states displayed by samples with longer side chains (Figure 4D). This correlation is consistent with their higher crystallinity. As a result, in addition to thermal and mechanical properties, release profile of these bottlebrush elastomers can be tuned with network architecture without altering chemical composition, which significantly enhances their safety and biocompatibility. Cytocompatibility of PVL
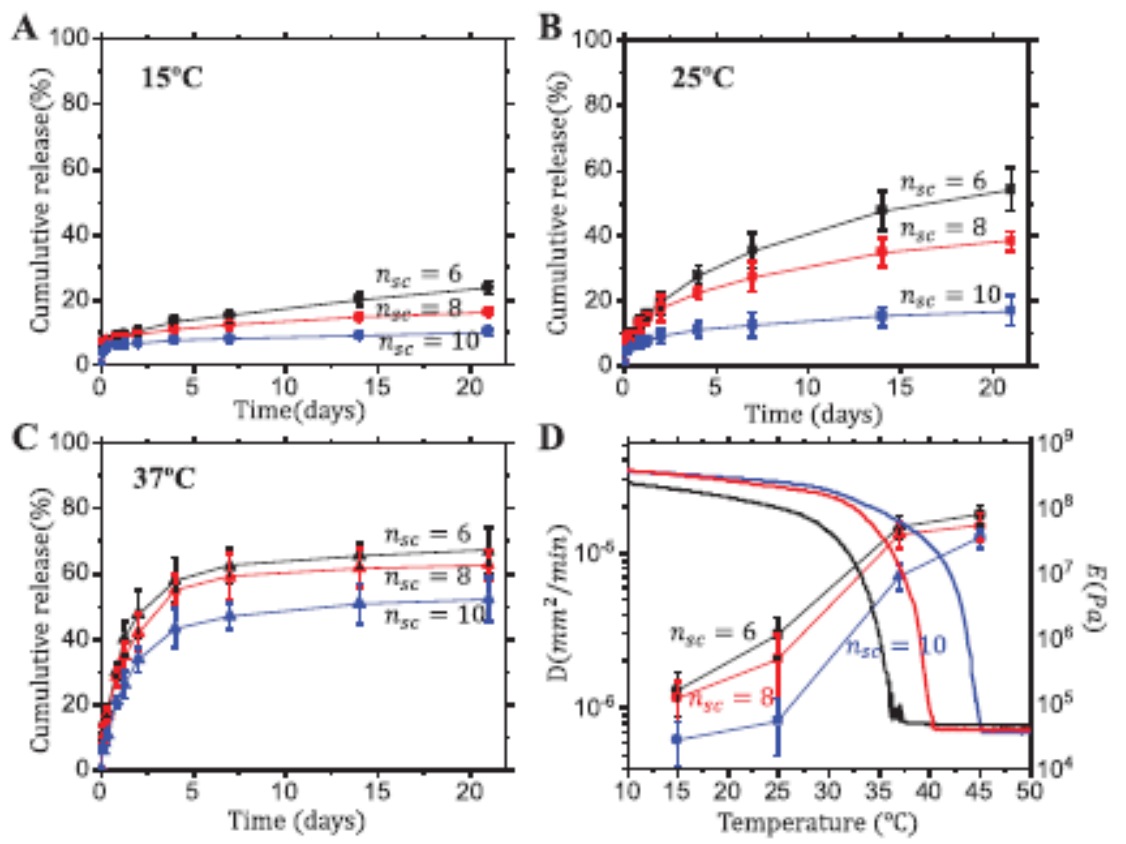

Figure 4. Controlled release. A-C) Rhodamine-B release profile of three PVL bottlebrush elastomers $(n x \square \square 50, n s c \square \square 6,8,10)$ at different temperatures as indicated. All samples have 
the same dimensions: discs with a thickness of $h \square \square 1 \mathrm{~mm}$ and diameter $d \square \square 8 \mathrm{~mm}$. The standard deviations from the mean are obtained by a series of independent measurements. D) Effective diffusion coefficients at different temperatures were obtained by fitting the corresponding release curves (A-C) with Equation 2 within a range of $M \mathrm{t} / M \infty \square \square \square 0.4$, where $M t$ and $M \infty \square$ are the cumulative amounts of rhodamine-B released at time $t$ and $\infty$, respectively. Corresponding DMA curves with the same $n \mathrm{sc}$ are added to show the concurrent softening transition.

bottlebrush elastomers is demonstrated by $>90 \%$ cell viability compared to the tissue culture plate as control (Figure 5A), adhesion and proliferation (Figure 5B,C) of NIH/3T3 mouse embryonic fibroblast cells cultured onto a series of samples with different $n \mathrm{sc}$. The combination of minimally invasive insertion capability, tissue mechanics replication, solvent-free composition, and enhanced biocompatibility profile provide PVL bottlebrush elastomers with a significant competitive edge over existing temperature-triggered drug-release matrices.[42] For example, current drug-delivery microneedles aim to reduce inflammation caused by mechanical mismatch between a rigid needle and soft dermis through soft coatings[43] or by being water soluble.[44] In contrast, a microneedle array made with PVL bottlebrush thermoplastic elastomers would be able to penetrate stiffer epidermis and become mechanically compliant with softer dermis while concurrently starting the release of small-molecule cargo into the body (Figure 5D). As a proofof- concept, we prepared $400 \mu \mathrm{m}$ long microneedles (to penetrate $\approx 100$ $\mu \mathrm{m}$ thick epidermis) loaded with rhodamine-B (Figure 5E). Even more severe issues are faced by intracortical implants that demonstrate most significant mechanical mismatch between soft brain tissue $(\approx 1 \mathrm{kPa})$ and electrodes $(\approx 10 \mathrm{MPa})$.[45] While some progress has been achieved with polymer composites with cellulose whiskers, $[14,46]$ bottlebrush elastomers afford superior brain tissue mechanical replication[ 13] and allow controlled release of antiforeign body reaction (FBR) drugs (Figure 5F). By incorporating crystallizable PVL side chains into bottlebrush network architecture, we demonstrate an unprecedented modulus change (104-106 times) upon melting. At ambient conditions these materials are hard $(0.1-1 \mathrm{Gpa})$ for effortless handling and storage, yet become as soft as tissue (103-105 Pa) in contact with temperatures relevant to the body $\left(28-43{ }^{\circ} \mathrm{C}\right)$. Therefore, these materials have great potential as implants as they are easily inserted into the body while minimizing adverse immune reaction caused by mechanical mismatch after implantation. Bottlebrush architecture allows for independently controlling the softening transition temperature and modulus in the soft state without changing its chemical composition, which makes them universally applicable for different organs from the cooler dermis $\left(\approx 33{ }^{\circ} \mathrm{C}\right)$ to warmer internal organs $\left(\approx 37^{\circ} \mathrm{C}\right)$ and from softer adipose tissue $(\approx 1 \mathrm{kPa})$ to harder tendons $(\approx 200 \mathrm{kPa})$. In addition, the bottlebrush architecture's programmability and solvent-free nature allows their use as matrices for controlled drug release. Difference in diffusion coefficients of the crystalline hard state versus amorphous soft state further enables stimuli-responsive release of small molecules, e.g., drugs, into the body. Concurrent softening and drug release upon insertion into the body have great potential for advanced implant applications such as reactive anti-FBR prosthetics, trans/sub-dermal drug-delivery devices, and carrying material for long-term in vivo probes, sensors or electronics and is readily expandable to other crystalline polyesters. 

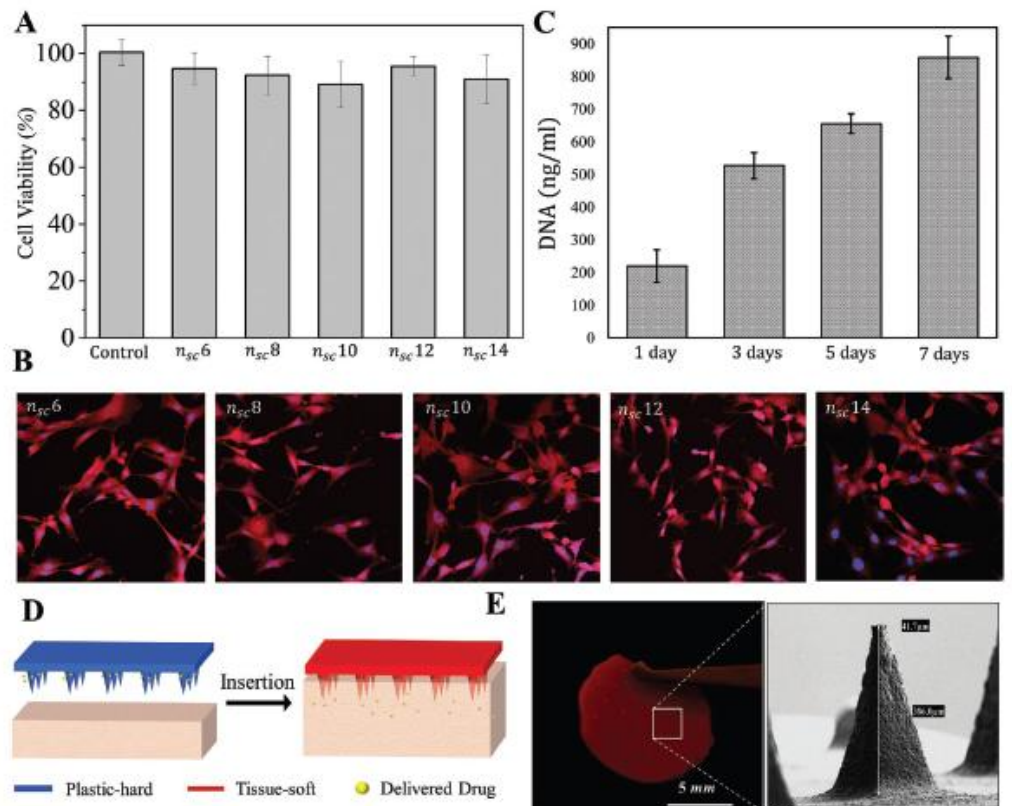

F
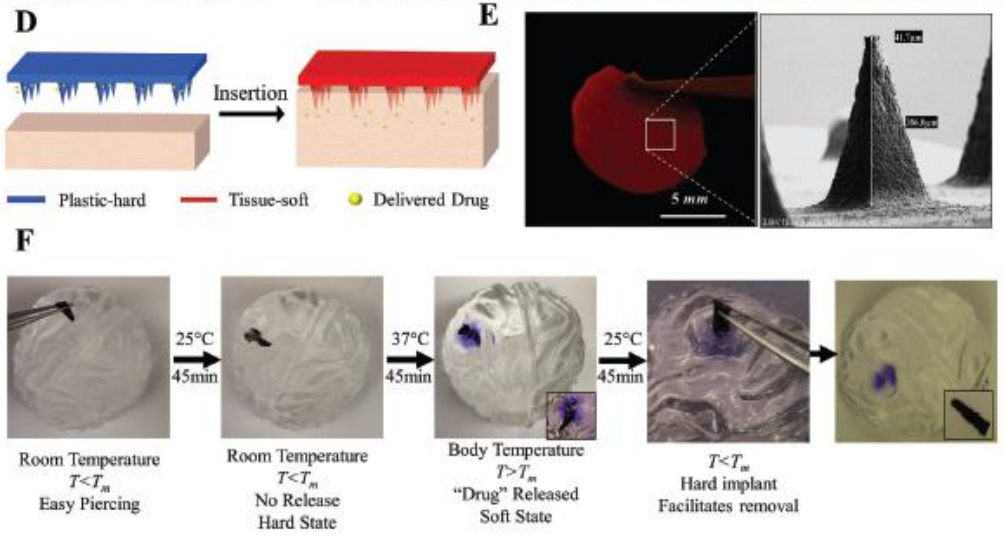

Figure 5. Biocompatibility and potential applications. A) Cell viability of NIH/3T3 embryonic fibroblast cells measured by analyzing the metabolic activity of cultured cells onto PVL bottlebrush elastomers ( $n x \square \square 100, n s c \square \square 6-\square 14$ ) ( $n \square \square 3$ ) after $24 \mathrm{~h}$ of incubation compared to the tissue culture plate as a control. B) Fluorescence microscopy images of actin-stained NIH/3T3 fibroblasts (ActinRed 555 and NucBlue) cultured onto different PVL bottlebrush elastomers after $3 \mathrm{~d}$. C) Quantified proliferation of NIH/3T3 cells cultured onto a PVL bottlebrush elastomer ( $n \mathrm{sc} \square \square 10, n x \square \square 100$ ) ( $n \square \square 3$ ) after 1, 3, 5, and $7 \mathrm{~d}$ measured by PicoGreen DNA assay.[41] D) Proposed application of PVL bottlebrush elastomers as transdermal drug release microneedle patches. E) Photo and SEM image of microneedle patch fabricated with PVL bottlebrush elastomers loaded with $0.1 \mathrm{wt} \%$ rhodamine-B as a sample drug. F) PVL bottlebrush elastomer shaped as a probe $(\approx 10 \square \square 2 \square \square 2 \mathrm{~mm})$ is loaded with a dye (crystal violet) imitating anti-FBR drugs and inserted into a tissue-mimicking polyacrylamide hydrogel under room temperature. While stable at room temperature, the "probe" starts to release the dye once temperature reaches $37 \square \mathrm{C}$. A stretching test shows the tissue-softness of PVL bottlebrush elastomer at this stage. After cooling, the "probe" becomes hard again to facilitate removal while the dye is left in the "brain" model.

\section{Experimental Section}

Materials: $\delta$-Valerolactone ( $\square 98 \%$ ) was obtained from Sigma-Aldrich and filtered to remove polymers. Tin(II) 2 ethylhexanoate( $\mathrm{Sn}(\mathrm{Oct}) 2 \square 95 \%)$, ethylene glycol anhydrous, 4dimethylaminopyridine (DMAP $\square 99 \%$ ), methacrylate chloride, methacrylate anhydride, phenylbis(2,4,6- trimethylbenzoyl)phosphineoxide (BAPOs), tetrahydrofuran (THF), toluene anhydrous, ethanol anhydrous, dichloromethane (DCM), triethylamine(TEA) were purchased from Aldrich and used as received, as were all other reagents and solvents.

Macromonomer Synthesis (DP $\square \square 10$ ): Ethanol (2.3 g, $50 \mathrm{mmol}), \delta$-valerolactone $(50 \mathrm{~g}, 500$ $\mathrm{mmol}), 50 \mathrm{~mL}$ anhydrous toluene was added in an oven-dried flask. To the solution was added 3 A molecular sieves and the mixture was dried for $48 \mathrm{~h}$. The solution was filtered into a $200 \mathrm{~mL}$ 
round bottom flask. Tin(II) 2-ethylhexanoate $(1 \mathrm{~g})$ was added via syringe. The reaction mixture was heated to $110 \square \mathrm{C}$ and aliquots were removed periodically and analyzed by H-NMR. Between 6 and $8 \mathrm{~h}$, the reaction became viscous and magnetic stirring became hard. After reaching to the degree of polymerization equal 10 , the reaction was cooled to room temperature. The contents were then poured into hexane to precipitate the polymer. The polymer precipitate was filtered, washed with methanol, air dried, and then further dried under reduced pressure.

The polymer $(55 \mathrm{~g}, \approx 50 \mathrm{mmol})$ was dissolved in $100 \mathrm{~mL}$ DCM and dried with anhydrous MgSO 4 overnight. The solution was filtered and transferred in a $200 \mathrm{~mL}$ oven-dried flask. TEA $(5.5 \mathrm{~g})$ was added to the flask and the temperature of mixture decrease to $5 \square \mathrm{C}$ using an ice bath and methacrylate chloride $(5 \mathrm{~g}, 55 \mathrm{mmol})$ was added dropwise to the mixture. The ice bath was removed and the temperature increased to $40 \square \mathrm{C}$. The reaction was continued for overnight. The contents were then concentrated and poured into hexane chilled in an ice bath to precipitate the polymer. The polymer precipitate was filtered, washed with methanol, air dried, and then further dried under reduced pressure. (1H NMR $(400 \mathrm{MHz}, \mathrm{CDCl} 3, \delta): 1.23(\mathrm{t}, 3 \mathrm{H} ; \mathrm{CH} 3 \square \mathrm{CH} 2 \square \mathrm{O}-$ ); 1.91(s, 3H; $\square \mathrm{C}(\mathrm{CH} 3) \square \mathrm{CH} 2) ; 1.63$ (m, 4nH; $\square \mathrm{COOCH} 2 \mathrm{CH} 2 \mathrm{CH} 2 \mathrm{CH} 2 \mathrm{COO} \square, \mathrm{PVL}) ; 2.31$ (m, 2nH; $\square \mathrm{COOCH} 2 \square, \mathrm{PVL}$ ); 3.21(q, 2H; CH3 $\square \mathrm{CH} 2 \square \mathrm{O} \square$ ); 4.09 (m, 2nH; $\square \mathrm{CH} 2 \mathrm{OOC} \square$, PVL); 6.06, 5.55 (s, H, H; $\square \mathrm{C}(\mathrm{CH} 3) \square \mathrm{CH} 2$ ); MW $\square \square 1100$ from NMR)

Cross Linker Synthesis: (DP $\square \square 35$ ). Dried ethylene glycol (1.0 g, $16 \mathrm{mmol}$ ), $\delta$-valerolactone (56 $\mathrm{g}, 560 \mathrm{mmol}), 50 \mathrm{~mL}$ anhydrous toluene was added in an oven-dried flask. To the solution was added 3 A molecular sieves and the mixture was dried for $48 \mathrm{~h}$. The solution was filtered into a $200 \mathrm{~mL}$ round bottom flask. Tin(II) 2-ethylhexanoate $(1 \mathrm{~g})$ was added via syringe. The reaction mixture was heated to $110 \square \mathrm{C}$ and aliquots were removed periodically and analyzed by H NMR. After $12 \mathrm{~h}$ the reaction completes with $\approx 95 \%$ conversion and degree of polymerization 35 , the reaction was cooled to room temperature. The contents were then poured into hexane chilled in an ice bath to precipitate the polymer. The polymer precipitate was filtered, washed with methanol, air dried, and then further dried under reduced pressure.

The polymer (56 g, $\approx 16 \mathrm{mmol}$ ) was dissolved in $100 \mathrm{~mL}$ DCM and dried with anhydrous MgSO4 overnight. The solution was filtered and transferred in a $200 \mathrm{~mL}$ oven-dried flask. DMAP ( $2 \mathrm{~g}$ ) was added to the flask and the temperature of mixture decrease to $5 \square \mathrm{C}$ using ice bath and methacrylate anhydride $(3.7 \mathrm{~g}, 24 \mathrm{mmol}$ ) was added dropwise to the mixture. The ice bath was removed and the temperature increased to $40 \square \mathrm{C}$. The reaction was continued for overnight. The contents were then concentrated and poured into hexane chilled in an ice bath to precipitate the polymer. The polymer precipitate was filtered, washed with methanol, air dried, and then further dried under reduced pressure. $(1 \mathrm{H} \mathrm{NMR}(400 \mathrm{MHz}, \mathrm{CDCl} 3, \delta): 1.91(\mathrm{~s}, 6 \mathrm{H}$; $\square \mathrm{C}(\mathrm{CH} 3) \square \mathrm{CH} 2) ; 1.63(\mathrm{~m}, 4 \mathrm{mH}, \square \mathrm{COOCH} 2 \mathrm{CH} 2 \mathrm{CH} 2 \mathrm{CH} 2 \mathrm{COO} \square$; PVL); 2.31 (m, 2mH, $\square \mathrm{COOCH} 2-; \mathrm{PVL}) ; 3.68(\mathrm{t}, 4 \mathrm{H} ; \square \mathrm{O} \square \mathrm{CH} 2 \square \mathrm{CH} 2 \square \mathrm{O} \square$ );4.09 (m, 2mH; $\square \mathrm{CH} 2 \mathrm{OOC} \square, \mathrm{PVL}$ ); 6.06, 5.55 (s, 2H,2H; $\square \mathrm{C}(\mathrm{CH} 3) \square \mathrm{CH} 2)$. MW $\square \square 4200$ from NMR)

Bottlebrush PVL Elastomer Films: All bottlebrush elastomers were prepared by one-step copolymerization of macromonomer (1) with different molar ratios of cross-linker (2). The initial reaction mixtures contained: $\approx 60 \mathrm{wt} \%$ macromonomers $(\mathbf{1}), 1.5 \mathrm{wt} \%$ BAPOs photoinitiator, and $\approx 40 \mathrm{wt} \%$ toluene as solvent. For rhodamine-B release experiments, desired concentration of rhodamine- $\mathrm{B}$ is added at this stage to accurately control its total amount. First, the mixtures were degassed by bubbling dry $\mathrm{N} 2$ for $30 \mathrm{~min}$. Then, to prepare films, the mixtures were injected between two glass plates with a $2.3 \mathrm{~mm}$ poly(dimethylsiloxane) (PDMS) spacer and polymerized at room temperature for $12 \mathrm{~h}$ under $\mathrm{N} 2$ using a UV cross-linking chamber (365 nm UV lamp, 0.1 $\mathrm{mW} \mathrm{cm}-2,10 \mathrm{~cm}$ distance). Films were washed with THF (2 $\square \square$ with enough to immerse and fully swell the films, each time for $8 \mathrm{~h}$ ) in glass Petri dishes. The samples were then deswelled with ethanol and dried in a $75 \square \mathrm{C}$ oven. The conversion of monomers to elastomers (gel fraction) was between 75 to $98 \mathrm{wt} \%$ depending on crosslinker concentration.

Temperature Dependence of Hard-to-Soft Elastomers: Dog bone-shaped samples with bridge dimensions of $12 \mathrm{~mm} \quad \square \square 2 \mathrm{~mm} \square \square \approx 1 \mathrm{~mm}$ were loaded into an RSA-G2 DMA (TA Instruments) and subjected to uniaxial oscillation under $1 \square \mathrm{C} \mathrm{min}-1$ increasing temperature at a 
constant frequency of $2 \mathrm{~Hz}$. From $\square 10 \square \mathrm{C}$ to its transition temperature, determined by DSC (TA Instruments Q200) in a typical heat/cool/heat run from $\square 80 \square \mathrm{C}$ to $60 \square \mathrm{C}$ at $10 \square \mathrm{C} \mathrm{min}-1$, the strain used was $0.05 \%$. Above transition temperature to $80 \square \mathrm{C}$, the strain used was $5 \%$. This increase is due to worse signal to noise ratio generated at low strain for much lower modulus. The accuracy of this measurement is verified by measuring modulus individually per $10 \square \mathrm{C}$ after $\square 20 \mathrm{~min}$ of resting at each temperature. The match between individual tests and the sweep test suggests equilibrium throughout the test.

True Stress-Elongation Measurements: Dogbone-shaped samples with bridge dimensions of 12 $\mathrm{mm} \square \square 2 \mathrm{~mm} \square \square \approx 1 \mathrm{~mm}$ were loaded into an RSA-G2 DMA (TA Instruments) and fully molten under $80 \square \mathrm{C}$. They were then subjected to uniaxial extension with constant strain rate of $0.1 \mathrm{~mm}$ $\mathrm{s}^{-1}$. Samples were stretched till rupture, revealing the entire mechanical profile.

$X$-ray Scattering Measurements and Analysis: Small-angle scattering experiments were performed using laboratory SAXS/WAXS machine (Xeuss from Xenocs) coupled to a GeniX 3D $\mathrm{CuK} \alpha$ generator $(\lambda 1.54 \mathrm{~A})$ and also at the ID02 beamline of the European Synchrotron Radiation Facility (ESRF) in Grenoble (France). The SAXS/WAXS measurements were performed in transmission geometry on thin diskshaped ( $8 \mathrm{~mm} \square \square 1 \mathrm{~mm}$ ) films. The recorded 2D scattering patterns were centered, calibrated, regrouped, and reduced to 1D using the SAXS utilities platform described elsewhere.[47] Analysis of diffraction data was performed using custom-designed software for Igor Pro environment (Wavemetrics Ltd.). For quantitative analysis of small-angle scattering curves the $1 \mathrm{D}$ correlation and interface distribution functions (IDF) were calculated as described previously.[48,49] To compute the WAXS crystallinity index, the experimental curves were fit to a sum of Gaussian functions representing each of the orthorhombic crystalline reflexes of the PVL lattice plus a broad peak (amorphous halo), which was modeled using Pearson VII functions. The crystallinity index was computed as a ratio of the surface under the crystalline reflexes to the total surface.[50]

Rhodamine-B Loaded Hard-to-Soft Elastomers and Its Release Profile:

$0.1 \mathrm{wt} \%$ rhodamine-B compared to macromonomer is dissolved in a series of elastomer precursor solution mentioned above for film synthesis with different $n$ sc. After same polymerization procedure, the resulting film appears pink instead of white. The films are then cut into identical disks with $8 \mathrm{~mm}$ radius and $1 \mathrm{~mm}$ thickness. While immersed in $10 \mathrm{~mL}$ aqueous solution with $\mathrm{pH} \square \square 7.4$ for 3 weeks under different temperature, aliquots are taken periodically, replaced with fresh solution and measured with UV-Vis spectroscopy (Biotek Instruments) to determine its concentration by absorption at $550 \mathrm{~nm}$. The amount of Rhodamine-B left in the elastomer can be approximated by deducting the dye released to water from the original amount.

Hard-to-Soft "Implant" Drug Release Preparation: Elastomer samples were immersed in 0.1 $\mathrm{wt} \%$ aqueous solution of acetaminophen at room temperature. The solution was then heated up to $80 \square \mathrm{C}$ to increase the loading rate. After $\approx 20 \mathrm{~h}$, the solution was allowed to cool down back to room temperature. The "implant" piece was then removed from the solution and appeared white after rinsing. The model "tissue" used is an acrylamide/bis-acrylamide hydrogel with $18 \mathrm{wt} \%$ solid content and 100:1 crosslinking density.

After gelation, the hydrogel is immersed in $1 \mathrm{~m} \mathrm{KOH} / 5 \mathrm{wt} \%$ o-cresol solution which chromatically detects acetaminophen. The resulting gel has a tissue-mimicking water content of $82 \mathrm{wt} \%$ and modulus of $80 \mathrm{kPa}$, which matches the modulus of Intervertebral disk posterior measured by compression along the axial direction (parallel to spine). For the release experiment, the hydrogel is removed from solution into a mineral oil bath for accurate control over temperature and avoiding vaporization of o-cresol. For similar experiment presented in Figure 5, $0.1 \mathrm{wt} \%$ crystal violet is used instead of acetaminophen and the hydrogel is used pristine without base treatment.

Cell Viability Assay: In vitro biological assessment of PVL bottlebrush elastomers ( $n x \square \square 100$, $n$ sc $\square \square 6,8,10,12,14)$ was performed using mouse NIH/3T3 embryonic fibroblast cells (American Type Culture Collection). The samples are melted slightly above $37 \square \mathrm{C}$ then cooled back down to $37 \square \mathrm{C}$ to ensure tissue-mimicking softness. The cells were cultured in Dulbecco's 
modified Eagle medium (DMEM, Sigma-Aldrich) supplemented with $10 \%$ fetal bovine serum (FBS, Sigma-Aldrich), $2 \square \square 10 \square 3 \mathrm{~m} \mathrm{l-glutamine}$ and $1 \%$ penicillin-streptomycin (SigmaAldrich) and incubated in a humidified incubator at $37 \square \mathrm{C}$ with $5 \% \mathrm{CO} 2$. A cell suspension was prepared by treating the cultured NIH/3T3 fibroblasts with trypsin-EDTA solution (SigmaAldrich) for $5 \mathrm{~min}$. After neutralizing the enzyme and centrifugation, the cells were suspended in fresh DMEM medium. Afterward, the prepared cells suspension at a density of 105 cells mL $\square 1$ was added to sterilized PVL elastomers samples in 24-well culture plates. The PVL samples were submerged in $70 \%$ ethanol for $30 \mathrm{~min}$, then rinsed three times with potassium phosphate buffer solution (PBS) for $15 \mathrm{~min}$. This procedure was repeated five times. The PVL samples were then dried overnight under a biosafety cabinet. The tissue culture polystyrene wells were served as the control. The metabolic activity of cultured cells of the PVL bottlebrush elastomers was tested after $24 \mathrm{~h}$ incubation by means of PrestoBlue assay (Invitrogen). PrestoBlue reagent was added to each well of the culture plate containing cells with PVL bottlebrush elastomers ( $n x$ $\square \square 100, n \mathrm{sc} \square \square 6-\square 14$ ) and incubated for $30 \mathrm{~min}$. Then, fluorescence intensity was recorded at $560 / 590 \mathrm{~nm}$ (excitation/emission) using a microplate reader (Biotek Instruments) and compared with the control group. Results were presented as mean $\square \square$ standard deviation of the relative cell viability to the control group.

Fluorescence Microscopy: The NIH/3T3 cells on substrate was monitored by fluorescence microscopy. A cell suspension at a density of 105 cells mL $\square 1$ cultured on PVL bottlebrush elastomers ( $n x \square \square 100, n$ sc $\square \square 6-\square 14$ ) and incubated for $3 \mathrm{~d}$ in a humidified incubator at 37 $\square \mathrm{C}$ with 5\% CO2. Cells were fixed with $4 \%$ paraformaldehyde and stained with ActinRed 555 and NucBlue (Invitrogen), the fluorescence imaging was performed using a laser scanning confocal microscope (Olympus).

Cell Proliferation Assay: In order to evaluate cells proliferation, the PVL bottlebrush elastomer ( $n x \quad \square \square 100, n$ sc $\square \square 10$ ) samples were placed in a 24-well plate, and then NIH/3T3 cells suspension at a density of 105 cells $\mathrm{mL} \square 1$ were cultured onto them. Cell proliferation on substrates was quantified measuring the DNA contents ( $n \square \square 3$ ) by means of Quanti-iT PicoGreen dsDNA kit (Invitrogen) following the manufacturer's protocol.

Microneedle Fabrication: In order to fabricate microneedle patches, disc-shaped PVL networks (diameter: $8 \mathrm{~mm}$, thickness: $1 \mathrm{~mm}$ ) were incubated at $45 \square \mathrm{C}$ for $1 \mathrm{~h}$. Then, the bottlebrush elastomers were inserted onto a silicone microneedle template (MPatch, Micropoint Technologies) and incubated at $45 \square \mathrm{C}$ under compression overnight. Subsequently, the cast bottlebrush elastomers were incubated at room temperature for $24 \mathrm{~h}$. Finally, the microneedle patches were gently removed from the silicone template.

\section{Acknowledgements}

The authors acknowledge funding from the National Science Foundation (DMR 1921835, DMR 1436201 and DMR 2004048). D.A.I. and E.B. acknowledge the Ministry of Science and Higher Education of Russian Federation for financial support in the frame of project RFME-19 $\square \square$ 0100, N 075-15-2019-1889 from December 5, 2019. The work was done in the frame of state task № 0074-2019-0014 (registration № AAAA-A19- 119101590029-0). The authors acknowledge perfect technical support of the personnel of the ID02 beamline of the European Synchrotron Radiation Facility (ESRF) in Grenoble, France.

\section{Conflict of Interest}

The authors declare no conflict of interest.

\section{Author Contributions}

D.Z. and E.D. contributed equally to this work. D.Z. designed, synthesized, and characterized the monomers and crosslinkers. D.Z., X.H., and E.D. synthesized polymer networks. D.Z. performed thermal and mechanical tests and demonstrative drug release study. E.D. and F.F. performed quantitative measurement of drug release behavior and biological assays. M.V. and Q.L. proposed and concept-proofed the feasibility of a hardto- soft elastomer. E.A.B. and D.A.I. conducted X-ray studies and data analysis. S.S.S. is the principal investigator and corresponding author. D.Z. and S.S.S were primary writers of the manuscript. 


\section{Keywords}

bottlebrush elastomers, controlled release, network architecture, poly(valerolactone), stimuliresponsive materials

Received: August 5, 2020

Revised: October 22, 2020

Published online:

[1] S. Limmahakhun, A. Oloyede, K. Sitthiseripratip, Y. Xiao, C. Yan, Mater. Des. 2017, 114, 633.

[2] Y. Lu, D. Wang, T. Li, X. Zhao, Y. Cao, H. Yang, Y. Y. Duan, Biomaterials 2009, 30, 4143.

[3] I. R. Minev, P. Musienko, A. Hirsch, Q. Barraud, N. Wenger, E. M. Moraud, J. Gandar, M. Capogrosso, T. Milekovic, L. Asboth, R. F. Torres, N. Vachicouras, Q. Liu, N. Pavlova, S. Duis, A. Larmagnac, J. Vörös, S. Micera, Z. Suo, G. Courtine, S. P. Lacour, Science 2015, 347, 159.

[4] A. Carnicer-Lombarte, D. G. Barone, I. B. Dimov, R. S. Hamilton, M. Prater, X. Zhao, A. L. Rutz, G. G. Malliaras, S. P. Lacour, C. E. Bryant, J. W. Fawcett, K. Franze, Bioarxiv 2019, https://doi.org/10.1101/829648.

[5] S. P. Davis, B. J. Landis, Z. H. Adams, M. G. Allen, M. R. Prausnitz, J. Biomech. 2004, 37, 1155.

[6] J. Reeder, M. Kaltenbrunner, T. Ware, D. Arreaga-Salas, A. Avendano-Bolivar, T. Yokota, Y. Inoue, M. Sekino, W. Voit, T. Sekitani, T. Someya, Adv. Mater. 2014, 26, 4967.

[7] R. Reit, H. Abitz, N. Reddy, S. Parker, A. Wei, N. Aragon, M. Ho, A. Weittenhiller, T. Kang, M. Ecker, W. E. Voit, J. Mater. Chem. B 2016, 4, 5367.

[8] L. B. Kratchman, D. Schuster, M. S. Dietrich, R. F. Labadie, Audiol. Neurotol. 2016, 21, 244. [9] A. Richter, Y. Xie, A. Schumacher, S. Löeffler, R. Kirch, J. Al-Hasani, D. H. Rapoport, C. Kruse, A. Moser, V. Tronnier, S. Danner, U. G. Hofmann, Front. Neuroeng. 2013, 6, 6.

[10] C. S. Bjornsson, S. J. Oh, Y. A. Al-Kofahi, Y. J. Lim, K. L. Smith, J. N. Turner, S. De, B. Roysam, W. Shain, S. J. Kim, J. Neural Eng. 2006, 3, 196.

[11] I. Levental, P. C. Georges, P. A. Janmey, Soft Matter 2007, 3, 299.

[12] M. Vatankhah-Varnosfaderani, W. F. M. Daniel, M. H. Everhart, A. A. Pandya, H. Liang, K. Matyjaszewski, A. V. Dobrynin, S. S. Sheiko, Nature 2017, 549, 497.

[13] A. N. Keith, M. Vatankhah-Varnosfaderani, C. Clair, F. Fahimipour, E. Dashtimoghadam, A. Lallam, M. Sztucki, D. A. Ivanov, H. Liang, A. V. Dobrynin, S. S. Sheiko, ACS Cent. Sci. 2020, 6, 413.

[14] J. R. Capadona, K. Shanmuganathan, D. J. Tyler, S. J. Rowan, C. Weder, Science 2008, $319,1370$.

[15] M. Jorfi, M. N. Roberts, E. J. Foster, C. Weder, ACS Appl. Mater. Interfaces 2013, 5, 1517.

[16] J. R. McKee, S. Hietala, J. Seitsonen, J. Laine, E. Kontturi, O. Ikkala, ACS Macro Lett. 2014, 3, 266.

[17] F. (Kuo) Yang, A. Cholewinski, L. Yu, G. Rivers, B. Zhao, Nat. Mater. 2019, 18, 874.

[18] C. Min, W. Cui, J. Bei, S. Wang, Polym. Adv. Technol. 2005, 16, 608.

[19] G. Aktas Eken, M. H. Acar, Macromol. Chem. Phys. 2018, 219, 2.

[20] A. Merlettini, M. Gigli, M. Ramella, C. Gualandi, M. Soccio,

F. Boccafoschi, A. Munari, N. Lotti, M. L. Focarete, Biomacromolecules 2017, 18, 2499.

[21] W. F. M. Daniel, J. Burdyńska, M. Vatankhah-Varnoosfaderani, K. Matyjaszewski, J. Paturej, M. Rubinstein, A. V. Dobrynin, S. S. Sheiko, Nat. Mater. 2016, 15, 183.

[22] M. Rubinstein, R. H. Colby, Polymer Physics, Oxford University Press Inc., New York, USA 2003, pp. 274-281.

[23] D. Neugebauer, M. Theis, T. Pakula, G. Wegner, K. Matyjaszewski, Macromolecules 2006, $39,584$.

[24] S. Y. Yu-Su, S. S. Sheiko, H. Lee, W. Jakubowski, A. Nese, K. Matyjaszewski, D. Anokhin, D. A. Ivanov, Macromolecules 2009, 42, 9008. 
[25] C. R. López-Barrón, J. R. Hagadorn, S. J. Mattler, J. A. Throckmorton, Macromolecules 2020, 53, 3778.

[26] T. Uemura, N. Yanai, S. Watanabe, H. Tanaka, R. Numaguchi,

M. T. Miyahara, Y. Ohta, M. Nagaoka, S. Kitagawa, Nat. Commun. 2010, 1, 83.

[27] S. Nojima, Y. Ohguma, S. Namiki, T. Ishizone, K. Yamaguchi, Macromolecules 2008, 41, 1915.

[28] Q. Li, J. Zhou, M. Vatankhah-Varnoosfaderani, D. Nykypanchuk, O. Gang, S. S. Sheiko, Macromolecules 2016, 49, 1383.

[29] A. A. Yevstropov, B. V. Lebedev, T. G. Kulagina, N. K. Lebedev, Polym. Sci. U.S.S.R. 1982, 24, 628.

[30] S. S. Sheiko, A. V. Dobrynin, Macromolecules 2019, 52, 7531.

[31] M. Aubin, R. E. Prud'homme, Polymer 1981, 22, 1223.

[32] C. Clair, A. Lallam, M. Rosenthal, M. Sztucki, M. Vatankhah- Varnosfaderani, A. N. Keith, Y. Cong, H. Liang, A. V. Dobrynin, S. S. Sheiko, D. A. Ivanov, ACS Macro Lett. 2019, 8, 530.

[33] L. Tang, J. W. Eaton, Am. J. Clin. Pathol. 1995, 103, 466.

[34] N. U. Zitzmann, T. Berglundh, J. Clin. Periodontol. 2008, 35, 286.

[35] A. K. Alderman, E. G. Wilkins, H. M. Kim, J. C. Lowery, Plast. Reconstr. Surg. 2002, 109, 2265.

[36] F. Le Devedec, H. Boucher, D. Dubins, C. Allen, Mol. Pharmaceutics 2018, 15, 1565.

[37] R. Jalil, J. R. Nixon, J. Microencapsulation 1990, 7, 297.

[38] M. Hedenqvist, U. W. Gedde, Prog. Polym. Sci. 1996, 21, 299.

[39] M. C. Chen, M. H. Ling, K. W. Wang, Z. W. Lin, B. H. Lai, D. H. Chen, Biomacromolecules 2015, 16, 1598.

[40] J. Siepmann, F. Siepmann, J. Controlled Release 2012, 161, 351.

[41] M. Sonnaert, I. Papantoniou, F. P. Luyten, J. Schrooten, Tissue Eng., Part C 2015, 21, 519.

[42] N. Kamaly, B. Yameen, J. Wu, O. C. Farokhzad, Chem. Rev. 2016, 116, 2602.

[43] H. S. Gill, M. R. Prausnitz, J. Controlled Release 2007, 117, 227.

[44] M. Wang, L. Hu, C. Xu, Lab Chip 2017, 17, 1373.

[45] A. E. Rochford, A. Carnicer-Lombarte, V. F. Curto, G. G. Malliaras, D. G. Barone, Adv. Mater. 2020, 32, 1903182.

[46] J. P. Harris, J. R. Capadona, R. H. Miller, B. C. Healy, K. Shanmuganathan, S. J. Rowan, C. Weder, D. J. Tyler, J. Neural Eng. 2011, 8, 066011.

[47] M. Sztucki, T. Narayanan, J. Appl. Crystallogr. 2006, 40, s459.

[48] O. Glatter, O. Kratky, Small Angle X-ray Scattering, Academic Press Inc. Ltd., London, UK 1982.

[49] N. Stribeck, W. Ruland, J. Appl. Crystallogr. 1978, 11, 535.

[50] D. A. Ivanov, G. Bar, M. Dosiere, M. H. J. Koch, Macromolecules 2008, 41, 9224. 